Cómo citar: Ferrero F. Discontinuación de investigaciones en pediatría. Arch Argent Pediatr 2017;115(4):308-309.

\section{REFERENCIAS}

1. Scherer RW, Langenberg P, Von Elm E. Full publication of results initially presented in abstracts. Cochrane Database Syst Rev 2007:(2):MR000005.

2. Pica N, Bourgeois F. Discontinuation and nonpublication of randomized clinical trials conducted in Children. Pediatrics 2016;138(3):e20160223.

3. De Angelis CD, Drazen JM, Frizelle FA, et al. Clinical trial registration: a statement from the International Committee of Medical Journal Editors. JAMA 2004;292(11):1363-4.

4. Dal-Ré R, Ioannidis JP, Bracken MB, et al. Making prospective registration of observational research a reality. Sci Transl Med 2014;6(224):224cm1.

5. Kasenda B, Von Elm E, You J, et al. Prevalence, characteristics, and publication of discontinued randomized trials. JAMA 2014;311(10):1045-51.

6. Kasenda B, Von Elm EB, You J, et al. Learning from failure-rationale and design for a study about discontinuation of randomized trials (DISCO study). BMC Med Res Methodol 2012;12:131.

\title{
El principio de sincronicidad-equivalencia y la cronomedicina: su relevancia y exploración metodológica
}

\author{
The principle of synchronicity-equivalence and chronomedicine: relevance and \\ methodological exploration
}

\section{INTRODUCCIÓN}

Hace más de medio siglo, Carl Jung y Wolfgang Pauli propusieron una hipótesis revolucionaria: los fenómenos naturales estarían regidos no solo por el principio de causalidad, sino, además, por un tipo de orden natural que lograría interconectar y regular todos los eventos del mundo. Jung lo denominó "principio de sincronicidad o equivalencia" (SE), según fuese este percibido o no por la mente humana. ${ }^{1}$ En la actualidad, se conoce como "cronobiología" al efecto del principio de SE sobre el mundo natural, y como "cronomedicina", a sus efectos sobre los procesos de salud y enfermedad. La moderna metodología de la investigación cuenta con los medios necesarios para poder identificar aquellos patrones de ordenamiento, que, pese a su importancia, transcurren usualmente en forma imperceptible para la mente humana.

En el presente artículo, se describe en forma sucinta el origen, evolución e importancia del concepto de SE en la medicina moderna. ${ }^{2,3}$

\section{La causalidad: el principio conocido}

La causalidad consiste en una serie sucesiva de eventos, en la que el precedente (causa) origina al subsiguiente (efecto), dentro de los límites que le imponen el tiempo, el espacio y la lógica formal. ${ }^{1,2} \mathrm{Sin}$ embargo, la causalidad es, en realidad, una costumbre de la mente, nacida de un precedente histórico repetido, que origina una abstracción (recorte) producto de una mezcla de costumbres, creencias y sentido común, pues todo fenómeno no procede de una causa, sino de redes causales que nuestra falta de sutileza nos impide percibir plenamente. ${ }^{2}$ Por esta razón, la causalidad es solo aplicable a parcelas de realidad y no a la realidad completa o unicidad, en la que todo es, en algún grado, causa de todo (unus mundus). ${ }^{2}$

\section{La sincronicidad-equivalencia: el nuevo principio}

La sincronicidad consiste en la detección, por parte de la mente humana, de una coincidencia significativa y acausal entre dos o más sucesos del mundo. Es por esta razón que a este principio se lo conoce también como "coincidencia significativa" o "conexión acausal", debido a que el vínculo que establece entre los eventos en cuestión no es del tipo causa-efecto, ni tampoco azaroso, dado que su vinculación supera la probabilidad de ser explicable por azar. ${ }^{1}$ Más allá de la importancia de la sincronicidad como fenómeno psíquico, ${ }^{4}$ es decir, como la capacidad intuitiva de la mente humana de percibir dos fenómenos como vinculados significativa pero no causalmente, su valor radica en que pone de manifiesto la existencia de un principio de la naturaleza. ${ }^{5}$ La percepción de esta conexión no constituye una invención de la mente, sino la fugaz e intuitiva percepción del accionar de un patrón natural bajo el cual se ordenan, en dicho momento, diversos fenómenos del mundo, patrones oscilatorios que, si bien son omnipresentes, resultan habitualmente imperceptibles para el ser humano, quien solo puede percibirlos de modo fugaz (episodios sincronísticos). De ahí que Jung estableciera la distinción entre la existencia de dichos patrones de ordenamiento independientemente de su percepción por parte del hombre: principio de equivalencia (Gleichartigkeit), de la percepción de estos por parte de la mente humana: principio de sincronicidad (Sinngemässe Koinzidenz). ${ }^{5}$

Se ha postulado que la percepción de este principio de ordenamiento (equivalencia) en forma consciente (sincronicidad) sería posible gracias a la presencia de una dotación de reflejos localizados en sectores filogenéticamente antiguos del sistema nervioso central (sistema nervioso autónomopaleoencéfalo), destinados a poner en sintonía 
(consciente o inconsciente) al organismo con los ritmos del mundo, lo cual explicaría la existencia de los fenómenos sincronísticos en el ser humano, y los comportamientos colectivos coordinados de diversos seres vivos, tanto entre sí como con los ciclos de la Tierra. ${ }^{1,4,6,7}$ Cabe destacar que Arthur Schopenhauer ya había intuido la influencia del accionar coordinado de dos fuerzas naturales (causalidad y SE) en el desarrollo de los eventos del mundo, que metafóricamente asemejaba las localizaciones geográficas nacidas de la intersección entre meridianos y paralelos. ${ }^{1}$ Desde esta interpretación, los fenómenos estarían sujetos a un orden generativo causal (linealidad), el cual se desarrollaría bajo la influencia de un patrón de ordenamiento cíclico reinante en dicho momento y lugar (complejidadrecursividad) ${ }^{1,2,6,8}$

\section{El principio de sincronicidad-equivalencia y la cronobiología}

El universo está sujeto a ritmos y ciclos, los cuales, junto con la fuerza de la gravedad y la composición química de la atmósfera terrestre, han guiado el curso evolutivo de la vida. Algunos de estos ritmos (relojes exógenos) han llegado a inducir ritmos endógenos (relojes biológicos) en los organismos, lo que contribuyó a su adaptación al medio, fenómeno conocido como "cronobiología". Surgieron, entonces, ritmos endógenos basados en la duración del día (20-28 horas) o circadianos, mayores de 20 horas (ultradianos) o menores de 28 horas (infradianos). ${ }^{9}$ Existen en el hombre, al menos, unos 180 ritmos, con sus relojes biológicos localizados no solo en el sistema nervioso (núcleo supraquiasmático hipotalámico, etc.), sino también en las vísceras (hígado, corazón, pulmón, etc.).

Sin embargo, debe tenerse presente la posible existencia de ciclos cuyos ritmos podrían ser de décadas o siglos y, por ende, carentes de relojes internos y, por consiguiente, de difícil identificación, lo cual no descarta que pudiesen ser de influencia clave en el funcionamiento del organismo (cronofisiología), el desarrollo de sus enfermedades (cronopatología) y su respuesta a la terapéutica (cronofarmacología). ${ }^{9}$

\section{La cronomedicina y su exploración metodológica}

En pos del descubrimiento de estos patrones ocultos de ordenamiento, una herramienta pertinente sería la data mining. Esta se dedica, particularmente, a la detección de los patrones subyacentes en los conjuntos de datos. Estos patrones revelan asociaciones que, de otra forma, permanecerían ocultas, pero que, sin embargo, solo cobran valor si un intérprete logra otorgarles un sentido (coincidencia significativa), y pueden únicamente llegar a ser consideradas como causales en tanto esto sea documentado por otros métodos estadísticos. Si bien, durante este procedimiento, hay hipótesis implícitas, por ejemplo, durante la selección del conjunto de datos por explorar, estas no se explicitan a priori. La data mining, en el mejor de los casos, revela un patrón interesante en los datos, que es de utilidad en tanto y en cuanto se le encuentre un sentido. ${ }^{10}$

\section{CONCLUSIÓN}

La SE sería un principio general de la naturaleza, complementario a la causalidad, capaz de infundirles patrones de ordenamiento cíclico a los fenómenos del mundo. Este principio estaría implicado en la existencia de los ritmos fisiológicos (cronobiología) y fisiopatológicos (cronopatología). La percepción consciente y transitoria de su existencia se conoce como evento sincronístico, pero la dificultad de su percepción torna indispensable el uso de metodología apropiada para tal fin, como es el caso de la data mining. Resulta crucial, entonces, lograr una mayor comprensión de las características y dinámica de este principio a fin de alcanzar una mejor comprensión de su influencia sobre la salud, la enfermedad y la terapéutica.

Dr. Carlos G. Musso ${ }^{a}$, estudiante Francisco Baez, estudiante María J. Elias ${ }^{a}$ y Dra. Mónica Schpilberg ${ }^{b}$.

a. Departamento de Fisiología, Instituto Universitario del Hospital Italiano de Buenos Aires, Argentina.

b. Departamento de Investigación del Hospital Italiano de Buenos Aires, Argentina.

http:/ / dx.doi.org/10.5546/ aap.2017.309

Texto completo en inglés:

http:/ / dx.doi.org/10.5546/ aap.2017.eng.309

Cómo citar: Musso CG, Baez F, Elias MJ, et al. El principio de sincronicidadequivalencia y la cronomedicina: su relevancia y exploración metodológica. Arch Argent Pediatr 2017;115(4):309-310.

\section{REFERENCIAS}

1. Jung CG. Sincronicidad. Málaga: Sirio, 1988.

2. PeatFD.Sincronicidad.Puenteentrementey materia. Barcelona: Kairós, 1988

3. Verene DP. Coincidence, historical repetition, and selfknowledge:Jung, Vico, and Joyce. J Anal Psychol 2002; 47(3):45978.

4. Yiassemides A. Chronos in synchronicity: manifestations of the psychoid reality. J Anal Psychol 2011;56(4):451-70.

5. Giegerich W. A serious misunderstanding: synchronicity and the generation of meaning. J Anal Psychol 2012;57(4):500-11.

6. Combray J. Synchronicity. Nature and Psyche in an Interconnected Universe. Texas: A\&M University Press, 2009.

7. Slater VE. Modern physics, synchronicity and intuition. Holist Nurs Pract 1992;6(4):20-5.

8. Musso CG, Bezic J, ChristiansenS, et al. Histological glomerular patterns as chaotic attractors. Electron J Biomed 2007;1:21-7.

9. Tamargo J, Barberá J. Cronobiología, farmacología y patología. Barcelona: Mayo, 2005.

10. Dawson-Saunders B, Trapp R. Bioestadística médica. México: Manual Moderno, 1993:51-2. 